Pacific Journal of Mathematic 


\title{
EXTENSIONS OF CONTINUOUS AFFINE FUNCTIONS
}

\section{LEONARD ASIMOW}

\begin{abstract}
Conditions are given for a closed face $F$ of a compact convex set $X$ to have the property that if $f \in A(F), g_{1}, \ldots$, $g_{m} \in A(X)$, and $f$ dominates each $g_{i}$ on $F$ then $f$ can be extended to $g \in A(X)$ where $g$ dominates each $g_{i}$ on $X$.
\end{abstract}

Let $X$ be a compact convex set in a Hausdorff locally convex space. We identify $X$ in the standard fashion with the set of positive elements of norm one in $A(X)^{*}$ (weak*-topology), where $A(X)$ is the ordered Banach space (sup-norm) of continuous affine functions on $X$. A face of $X$ is a convex subset which contains the endpoints of every open line segment in $X$ which it intersects. It is known (for example [2]) that every continuous affine function on a closed face $F$ of $X$ admits a continuous affine extension to all of $X$ if and only if the linear span, $\langle F\rangle$, of $F$ is weak* closed in $A(X)^{*}$. If additional conditions of a geometric nature on $F$ and $X$ are made then much more can be said about the type of extensions which are possible. For example if $X$ is a Choquet simplex (in which case $\langle F\rangle$ is weak* closed whenever $F$ is), a theorem of Edwards [3] states that

$\left(^{*}\right)$ if $\left\{f_{i}\right\}_{i=1}^{m},\left\{g_{j}\right\}_{j=1}^{n} \in A(X)$ and $f \in A\left(F^{\prime}\right)$ such that

$$
\left.f_{i}\right|_{F} \leqq f \leqq\left. g_{j}\right|_{F} \quad(i=1, \cdots, m ; j=1, \cdots n)
$$

then there is an extension $g \in A(X)$ of $f$ such that

$$
f_{i} \leqq g \leqq g_{j} \quad(i=1, \cdots, m ; j=1, \cdots, n) .
$$

This extension property is quite strong in the sense that it in fact characterizes simplexes among the compact convex sets.

One can ask under what conditions on $F$ and $X$ the following weaker extension property holds:

$\left({ }^{*}\right)$ if $\left\{f_{i}\right\}_{i=1}^{m} \in A(X)$ and $f \in A(F)$ such that

$$
\left.f_{i}\right|_{F} \leqq f \quad(i=1, \cdots, m)
$$

then there is an extension $g \in A(X)$ of $f$ such that

$$
f_{i} \leqq g \text {. }
$$

Closed faces which possess property $\left(^{* *}\right)$ are termed strongly archimedean by Alfsen [1] (see also Størmer [5] for the origin of the terminology). In [2] we give conditions on $F$ such that $\left({ }^{* *}\right)$ holds for functions $f_{i}, f$ identically zero on $F$. This implies in particular that $F$ is (within a $G_{o}$ set) a peak-face of $X$. We give here a some- 
what strengthened form of these conditions which guarantees an extension can be found such that $\left({ }^{* *}\right)$ holds in general.

We shall say $X$ is decomposable at the closed face $F$ (under $f$ ) if there exists a bounded linear functional $f$ on $A(X)^{*}$ such that $f$ is identically zero on $\langle F\rangle^{-}$(weak* closure) and $X=$ conv $(K \cup F)$, where $K=\{x \in X: 1 \leqq f(x) \leqq\|f\|\}$. If $X$ is decomposable at $F$ and the linear span of $F$ is weak* closed, then we show (Theorem 2.6 and Corollary 2.7) that the extension property $\left({ }^{* *}\right)$ holds.

The closed faces of $\alpha$-polytopes (see Phelps [4]), for example, satisfy these conditions and hence are strongly archimedean. We show also that if $F$ is a closed face complemented in $X$ then a weak version of the extension property $\left({ }^{*}\right)$ can be obtained. As a corollary we obtain $\left(^{*}\right)$ as stated for simplexes.

1. Preliminaries. Let $h$ be any real-valued function on the compact convex set $X$. We call the set of ordered pairs $(x, r) \in X \times$ $R \subset A(X)^{*} \times R$ such that $r \geqq h(x)$ the upper-graph of $h$. The lowergraph is defined analogously. We note that $h$ is convex if and only if upper-graph $(h)$ is convex and $h$ is concave if and only if lowergraph $(h)$ is convex. Also $h$ is lower-semi-continuous if and only if upper-graph $(h)$ is closed and upper-semi-continuous if and only if lower-graph $(h)$ is closed.

If $x \in X$ we define the gage functional $p_{x}$ on $X$ by

$$
p_{x}(y)=\inf \{r \geqq 0: y \in x+r(X-x)\} .
$$

Then $p_{x}$ is lower-semi-continuous, convex and affine along any line segment in $X$ with one endpoint $x$. Also for each $y \in X$ there is a $z \in X$ such that

$$
y=p_{x}(y) z+\left(1-p_{x}(y)\right) x .
$$

Let $F$ be a closed face of $X$.

Proposition 1.1. For each $y \in X$ the function $x \rightarrow p_{x}(y)$ is lowersemi-continuous on $F$.

Proof. Let $\varphi: F \times X \times[0,1] \rightarrow X$ be defined by

$$
\varphi(x, z, \lambda)=\lambda z+(1-\lambda) x .
$$

Then for $x \in F, p_{x}(y) \leqq r$ if and only if there is a $z \in X$ and $s \in[0, r]$ such that

$$
y=s z+(1-s) x .
$$

Thus $\left\{x \in F: p_{x}(y) \leqq r\right\}$ is exactly the natural projection of $\varphi^{-1}(y) \cap$ 
$(F \times X \times[0, r])$ into $F$ which is clearly closed.

We define $p_{F}: X \rightarrow[0,1]$ by

$$
p_{F}(y)=\inf \left\{p_{x}(y): x \in F\right\} \text {. }
$$

By the proposition the infimum is actually attained and so each $y \in X$ can be written as

$$
y=p_{F}(y) z+\left(1-p_{F}(y)\right) x ; \quad z \in X \text { and } x \in F .
$$

It also follows that $p_{F}$ is affine along the line segment $[x, z]$ and thus $p_{F}(z)=1$. In addition upper-graph $\left(p_{F}\right)$

$$
=\{(x, r): x \in X, r \geqq 1\} \cup \operatorname{conv}[(X \times\{1\}) \cup(F \times\{0\})],
$$

and hence is a closed convex set. Consequently $p_{F}$ is lower-semicontinuous and convex.

In the sequel it is necessary to assume that the closed face $F$ of $X$ is self-determining, that is, if $N$ is the weak* closure of $\langle F\rangle$ in $A(X)^{*}$ then $N \cap X=F$. If this is the case then let $q: A(X)^{*} \rightarrow$ $A(X)^{*} / N$ be the quotient map. The quotient space can be identified with the dual of the space of continuous affine functions on $q X$ vanishing at $0[2]$. We then define the semi-norm $p_{N}$ on $A(X)^{*}$ by

$$
p_{N}(x)=\|q x\| \text {. }
$$

It follows that $p_{N}$ is weak* lower-semi-continuous and sub-additive on $A(X)^{*}$.

2. Decomposable faces. As our first step we give conditions which assure that if $h \in A(X),\left.h\right|_{F} \geqq 0$ then there is a $g \in A(X)$ such that $\left.g\right|_{F}=\left.h\right|_{F}$ and $g \geqq 0$ on $X$.

Lemma 2.1. Let $F$ be a subset of $X$ and assume there exists $M$ and $\beta(M \geqq 0$ and $0 \leqq \beta<1)$ such that if $h \in A(X),\left.h\right|_{F} \geqq 0, h+a \geqq 0$ on $X(a \geqq 0)$ there is a $g_{1} \in A(X),\left.g_{1}\right|_{F}=\left.h\right|_{F}, g_{1}+\beta a \geqq 0$ on $X$ and $\left\|g_{1}-h\right\| \leqq M a$.

Then for each $h \in A(X),\left.h\right|_{F} \geqq 0$, there is a $g \in A(X),\left.g\right|_{F}=\left.h\right|_{F}$, $g \geqq 0$ on $X$ and $\|g-h\| \leqq\|h\| /(1-\beta) M$.

Proof. Let $h \in A(X)$ such that $\left.h\right|_{F} \geqq 0$ be given. Then $h+\|h\| \geqq 0$ so there is $g_{1} \in A(X),\left.g_{1}\right|_{F}=\left.h\right|_{F}, g_{1}+\beta\|h\| \geqq 0$ and $\left\|g_{1}-h\right\| \leqq M\|h\|$. Now apply the hypothesis to $g_{1}$ with $a=\beta\|h\|$ and get $g_{2} \in A(X)$ such that $\left.g_{2}\right|_{F}=\left.h\right|_{F}, g_{2}+\beta^{2}\|h\| \geqq 0$ and $\left\|g_{2}-g_{1}\right\| \leqq \beta M\|h\|$. Continuing by induction we get a sequence $\left\{g_{n}\right\}_{n=1}^{\infty}$ such that $\left.g_{n}\right|_{F}=\left.h\right|_{F}$, $g_{n}+\beta^{n}\|h\| \geqq 0$ and $\left\|g_{n+1}-g_{n}\right\| \leqq \beta^{n} M\|h\|$. Thus $\left\{g_{n}\right\}_{n=1}^{\infty}$ converges uniformly to $g \in A(X)$ such that $\left.g\right|_{F}=\left.h\right|_{F}, g \geqq 0$ on $X$ and 


$$
\|g-h\| \leqq \sum_{n=1}^{\infty}\left\|g_{n+1}-g_{n}\right\|+\left\|g_{1}-h\right\| \leqq \frac{M}{1-\beta}\|h\| \text {. }
$$

THEOREM 2.2. Let $F$ be a self-determining face of $X$ and assume that $p_{F} \leqq \alpha p_{N}$. Then if $r>\alpha$ and $h \in A(X),\left.h\right|_{F} \geqq 0$, there exists $g \in A(X),\left.g\right|_{F}=\left.h\right|_{F}, g \geqq 0$ on $X$ and $\|h-g\| \leqq 2 r\|h\|$.

Proof. It suffices to show that the hypotheses of the preceding lemma is satisfied with $M=2$ and $\beta=1-1 / r$. Since $p_{N} \leqq p_{F} \alpha$ must be greater than or equal to one and hence $0 \leqq \beta<1$. Given $h \in A(X)$, $\left.h\right|_{F} \geqq 0$ and $h+a \geqq 0$ on $X(a>0)$ define $\bar{h}$ on $A(X)^{*}$ by

$$
\bar{h}=h+a p_{N} \text {. }
$$

Then $\bar{h}$ is sub-additive and weak* lower-semi-continuous. If $y \in X$ then since $y=p_{F}(y) z+\left(1-p_{F}(y)\right) x$ with $x \in F$ and $p_{F}(z)=1$ we have

$$
\begin{aligned}
\bar{h}(y)= & h(y)+a p_{N}(y)=p_{F}(y)\left(h(z)+a p_{N}(z)\right)+\left(1-p_{F}(y)\right) h(x) \\
& \geqq p_{F}(y)\left(h(z)+\frac{a}{\alpha}\right)+\left(1-p_{F}(y)\right) h(x) \\
= & p_{F}(y)(h(z)+a)+\left(1-p_{F}(y)\right) h(x)-a p_{F}(y)\left(1-\frac{1}{\alpha}\right) .
\end{aligned}
$$

Thus since $h(z)+a, h(x) \geqq 0$ and $\alpha<r$

$$
\bar{h}(y)+a \beta>0 \text { for all } y \in X \text {. }
$$

Let $Y=\operatorname{conv}(\{0\} \cup X)$ in $A(X)^{*}$. Then (*) continues to hold on $Y$. Thus $\left\{(x, r) \in A(X)^{*} \times R: r \geqq \bar{h}(x)+a \beta\right\}$ is a closed convex set disjoint from $Y \times\{0\}$. A weak* closed separating hyperplaneyields exactly the graph of a weak* continuous affine function $f$ on $A(X)^{*}$ such that $f>0$ on $Y, f<\bar{h}+a \beta$ on $A(X)^{*}$ and $0<f(0)<a \beta$. On $N$ we have $\bar{h}=h$ and hence $f<h+a \beta$ there. Since $N$ is a subspace and $h$ is linear we have $f=h+f(0)$ on $N$. Let $g=f-f(0)$. Then $g \in A(X),\left.g\right|_{F}=\left.h\right|_{F}$ and $g+a \beta>g+f(0)>0$ on $X$. Also

$$
g<f<\bar{h}+a \beta \leqq h+a\left(p_{N}+\beta\right) \text {. }
$$

Since $p_{N} \leqq 1$ on $\operatorname{conv}(X \cup-X)$ we have

$$
g<h+2 a \text { on } X \text { and }-X \text {. }
$$

Thus $\|h-g\| \leqq 2 a$ and the proof is complete.

In [2] we define the self-determining face $F$ to be conical in $X$ (under $f$ ) if $f$ is a bounded linear functional on $A(X)^{*}$ such that $f \equiv 0$ on $N=\langle F\rangle^{-}, f \geqq 0$ on $X$ and $x \in f(x) X+N$ for all $x \in X+N$. 
We will say that $X$ is decomposable at $F$ (under $f$ ) if $f$ is a bounded linear functional on $A(X)^{*}$ such that $f \equiv 0$ on $N$ and

$$
X=\operatorname{conv}(F \cup\{x \in X: 1 \leqq f(x) \leqq\|f\|\}) \text {. }
$$

Note that if $X$ is decomposable at $F$ then $F$ is automatically selfdetermining. Also, as noted in [2], if $X$ is decomposable at $F$ under $f$ then $F$ is a conical face of $X$ under $f$.

Proposition 2.3. If $X$ is decomposable at $F$ under $f$ then $p_{F} \leqq\|f\| p_{N}$.

Proof. If $y \in X$ then $y=\lambda z+(1-\lambda) x(0<\lambda<1)$ with $x \in F$, $z \in X$ and $f(z) \geqq 1$. Thus $p_{F}(y) \leqq \lambda$ and $f(y)=\lambda f(z) \geqq \lambda$ and hence

$$
p_{F} \leqq f \text {. }
$$

If $q: A(X)^{*} \rightarrow A(X)^{*} / N$ is the quotient map we can define $\bar{f}$ on $A(X)^{*} / N$ by $f=\bar{f} \cdot q$ (since $f \equiv 0$ on $N$ ). Also $\|f\|=\sup f(X)=$ $\sup \bar{f}(q X)=\|\bar{f}\|$ since the unit ball in $A(X) * / N$ is $\operatorname{conv}(q X \cup-q X)$. Hence for $y \in X$

$$
f(y)=\bar{f} \circ q(y) \leqq\|\bar{f}\|\|q y\|=\|\bar{f}\| p_{N}(y) .
$$

Thus

$$
f \leqq\|f\| p_{N} \text {. }
$$

Combining (1) and (2), $\quad P_{F} \leqq\|f\| p_{N}$.

COROLlaRy 2.4. If $X$ is decomposable at $F$ under $f$ then for any $h \in A(X),\left.h\right|_{F} \geqq 0$ and for any $r>\|f\|$ there is a $g \in A(X),\left.g\right|_{F}=\left.h\right|_{F}$, $g \geqq 0$ on $X$ and $\|g-h\| \leqq 2 r\|h\|$.

From $[2 ; \S 2]$, we have the following theorem.

THEOREM 2.5. If $X$ is decomposable at $F$ under $f$ then given $g_{1}, \cdots, g_{n} \in A(X)$, each $g_{i} \equiv 0$ on $F$, then there is an $h \in A(X), h \equiv 0$ on $F$ such that $h \geqq g_{1}, \cdots, g_{n}$ on $X$. Furthermore, if $r>\|f\|, h$ can be chosen such that $\|h\| \leqq r$.

By combining Theorem 2.5 and Corollary 2.4 we obtain a result of the type mentioned in the introduction.

THEOREM 2.6. If $X$ is decomposable at $F$ and $f_{1}, \cdots, f_{n}, h \in A(X)$ are given such that each $\left.f_{i}\right|_{F} \leqq\left. h\right|_{F}$ there is a $g \in A(X)$ such that $\left.g\right|_{F}=\left.h\right|_{F}$ and each $f_{i} \leqq g$ on $X$.

Proof. Since $\left.\left(h-f_{i}\right)\right|_{F} \geqq 0$ there is $g_{i} \in A(X),\left.g_{i}\right|_{F}=\left.\left(h-f_{i}\right)\right|_{F}$ and 
$g_{i} \geqq 0$ on $X$. Let $g_{i}^{\prime}=g_{i}+f_{i}$. Then $\left.g_{i}^{\prime}\right|_{F}=\left.h\right|_{F}$ and $g_{i}^{\prime} \geqq f_{i}$ on $X$. Now each $g_{i}^{\prime}-h$ is identically zero on $F$. Hence there is $g^{\prime} \in A(X)$, $g^{\prime} \equiv 0$ on $F$ and $g^{\prime} \geqq g_{i}^{\prime}-h$ on $X$. Let $g=g^{\prime}+h$. Then $\left.g\right|_{F}=\left.h\right|_{F}$ and

$$
g=g^{\prime}+h \geqq\left(g_{i}^{\prime}-h\right)+h=g_{i}^{\prime} \geqq f_{i} \quad(i=1, \cdots, n)
$$

on $X$.

Corollary 2.7. If $X$ is decomposable at the closed face $F$ and the linear span of $\langle F\rangle$ in $A(X)^{*}$ is weak* (or equivalently norm) closed, then for any $f_{1}, \cdots, f_{n} \in A(X)$ and $h \in A(F)$ such that each $\left.f_{i}\right|_{F} \leqq h$ there is an extension $g \in A(X)$ of $h$ such that each $f_{i} \leqq g$ on $X$.

Proof. Since $\langle F\rangle$ is closed we can find some extension $h^{\prime} \in A(X)$ of $h$ (see, for example [2], Th. 3.1). Thus, Theorem 2.6 applies with $f_{1}, \cdots, f_{n}$ and $h^{\prime}$.

3. Complemented faces. We shall say the closed face $F$ of $X$ is complemented in $X$ (by $F^{\prime}$ ) if there is a disjoint face $F^{\prime \prime}$ (not necessarily closed) in $X$ such that each $y \in X$ has a unique representation of the form

$$
y=\lambda x+(1-\lambda) z ; \quad x \in F, z \in F^{\prime} .
$$

This implies in particular that $\langle F\rangle$ and $\left\langle F^{\prime}\right\rangle$ are complemented subspaces in $A(X)^{*}$.

For a complemented face $F$ in $X$ we obtain a stronger extension property. We establish a preliminary result first.

Lemma 3.1. Let $F$ be a closed face in $X$ complemented by $F^{\prime \prime}$. Let $f \in A(F)$ and $g \in A(X)$ such that $f \leqq\left. g\right|_{F}$. Then the function $h$ defined by

$$
\begin{gathered}
h(y)=\lambda f(x)+(1-\lambda) g(z) ; \quad y=\lambda x+(1-\lambda) z \\
0 \leqq \lambda \leqq 1, x \in F, z \in F^{\prime \prime}
\end{gathered}
$$

is affine and lower-semi-continuous on $X$.

Proof. The fact that $h$ is affine follows directly from the definition of complemented faces. If $(y, h(y)) \in$ graph $(h)$ then

$$
\begin{aligned}
(y, h(y))=\lambda(x, f(x)) & +(1-\lambda)(z, g(z)) \\
& \in \operatorname{conv}(\operatorname{graph}(f) \cup \operatorname{graph}(g)) .
\end{aligned}
$$

If $(w, s) \in \operatorname{conv}(\operatorname{graph}(f) \cup \operatorname{graph}(g))$ then 


$$
\begin{gathered}
(w, s)=\alpha(x, f(x))+\beta\left(x^{\prime}, g\left(x^{\prime}\right)\right)+\gamma(z, g(z)) \\
\alpha+\beta+\gamma=1, x, x^{\prime} \in F, z \in F^{\prime}
\end{gathered}
$$

Since $g\left(x^{\prime}\right) \geqq f\left(x^{\prime}\right), s \geqq \alpha f(x)+\beta f\left(x^{\prime}\right)+\gamma g(z)=h(w)$ and hence $(w, s) \in$ upper-graph $(h)$. Thus

upper-graph $(h)=[\operatorname{conv}($ graph $(f) \cup \operatorname{graph}(g))] \cup$ upper-graph $(g)$

which is closed. Hence $h$ is lower-semi-continuous.

THEOREM 3.2. Let $F$ be a closed face complemented by $F^{\prime \prime}$ in $X$. Let $g_{i}, h_{j} \in A(X)$ such that there is $f \in A(F)$ and $f^{\prime}$ affine on $F^{\prime}$ with $\left.g_{i}\right|_{F} \leqq f \leqq\left. h_{j}\right|_{F}$ and $\left.g_{i}\right|_{F^{\prime}} \leqq f^{\prime} \leqq\left. h_{j}\right|_{F^{\prime}}(i=1, \cdots, m ; j=1, \cdots, n)$. Then for any $\varepsilon>0$ there is an extension $k \in A(X)$ of $f$ such that

$$
g_{i} \leqq k \leqq h_{j}+\varepsilon \quad(i=1, \ldots, m ; j=1, \cdots, n) .
$$

Proof. If $y \in X$ with $y=\lambda x+(1-\lambda) z\left(x \in F, z \in F^{\prime}\right.$ and $\left.0 \leqq \lambda \leqq 1\right)$ define $k_{0}(y)=\lambda f(x)+(1-\lambda) f^{\prime}(z)$. Then $k_{0}$ is affine and

$$
g_{i} \leqq k_{0} \leqq h_{j} \quad(i=1, \cdots, m ; j=1, \cdots, n) .
$$

Let $G=\operatorname{conv}\left(\bigcup_{i=1}^{m} \operatorname{graph}\left(g_{i}\right) \cup \operatorname{graph}(f)\right)$. Since $k_{0}$ is affine and $\left.k_{0}\right|_{F}=f$, graph $\left(g_{i}\right)$, graph $(f) \subset$ lower-graph $\left(k_{0}\right)$, a convex set. Hence

$$
G \subset \text { lower-graph }\left(k_{0}\right) \text {. }
$$

Similarly

$$
H \equiv \operatorname{conv}\left(\bigcup_{j=1}^{m} \operatorname{graph}\left(h_{j}\right) \cup \operatorname{graph}(f)\right) \subset \text { upper-graph }\left(k_{0}\right) \cdot
$$

Thus $G$ can be separated from $H+(0, \varepsilon / 2)$ by a hyperplane yielding $k_{1} \in A(X)$ such that

$$
\begin{gathered}
g_{i} \leqq k_{1} \leqq h_{j}+\frac{\varepsilon}{2} \quad(i=1, \cdots, m ; j=1, \ldots, n) \\
f \leqq\left. k_{1}\right|_{F} \leqq f+\frac{\varepsilon}{2} .
\end{gathered}
$$

Now define $\bar{k}_{1}(y)=\lambda f(x)+(1-\lambda) k_{1}(z)$ and as in the lemma $\bar{k}_{1}$ is affine lower-semi-continuous. Since $\left.g_{i}\right|_{F} \leqq f$ and $\left.\bar{k}_{1}\right|_{F}=f, G \subset$ lowergraph $\left(\bar{k}_{1}\right)$. Also $k_{1}-\varepsilon / 2 \leqq \bar{k}_{1}$ implies that $\operatorname{conv}\left(G \cup \operatorname{graph}\left(k_{1}-\varepsilon / 2\right)\right) \subset$ lower-graph $\left(\bar{k}_{1}\right)$. Since upper-graph $\left(\bar{k}_{1}\right)+(0, \varepsilon / 4)$ is closed, another separation yields $k_{2}$ such that

$$
\begin{gathered}
k_{1}-\frac{\varepsilon}{2}, g_{i} \leqq k_{2} \leqq \bar{k}_{1}+\frac{\varepsilon}{4} \leqq k_{1}+\frac{\varepsilon}{4} \leqq h_{j}+\frac{\varepsilon}{2}+\frac{\varepsilon}{4} \\
(i=1, \cdots, m ; j=1, \cdots, n) .
\end{gathered}
$$


and

$$
f \leqq\left. k_{2}\right|_{F} \leqq f+\frac{\varepsilon}{4}
$$

In particular $\left\|k_{2}-k_{1}\right\| \leqq \varepsilon / 2$. Continuing inductively we get a sequence $\left\{k_{r}\right\}_{r=1}^{\infty}$ such that

$$
\begin{gathered}
g_{i} \leqq k_{r} \leqq h_{j}+\sum_{s=1}^{r} \frac{\varepsilon}{2^{s}} \quad(i=1, \cdots, m ; j=1, \cdots, n) \\
f \leqq\left. k_{r}\right|_{F} \leqq f+\frac{\varepsilon}{2^{r}}
\end{gathered}
$$

and

$$
\left\|k_{r+1}-k_{r}\right\| \leqq \frac{\varepsilon}{2^{r}}
$$

Hence $\left\{k_{r}\right\}_{r=1}^{\infty}$ converges to $k \in A(X)$ such that

$$
g_{i} \leqq k \leqq h_{j}+\varepsilon \quad(i=1, \cdots, m ; j=1, \cdots, n)
$$

and

$$
\left.k\right|_{F}=f .
$$

Corollary 3.3. If $F$ is a closed face complemented in $X$ then for any $\varepsilon>0$ each $f \in A(F)$ has an extension $g \in A(X)$ such that

$$
\|g\| \leqq(1+\varepsilon)\|f\| \text {. }
$$

Corollary 3.4. (D. A. Edwards [3]). If $X$ is a simplex and $F$ is a closed face of $X$ with $g_{i}, h_{j} \in A(X)$ such that $g_{i} \leqq h_{j}(i=1, \cdots, m$; $j=1, \cdots, n)$ and $f \in A(F)$ such that $\left.g_{i}\right|_{F} \leqq f \leqq\left. h_{j}\right|_{F}$ then $f$ can be extended to $k \in A(X)$ such that $g_{i} \leqq k \leqq h_{j}(i=1, \ldots, m ; j=1, \cdots, n)$.

Proof. Since $X$ is a simplex $F$ has a complementary face $F^{\prime}$. Also the upper envelope $g^{\prime}$ of $g_{1} \vee \cdots \vee g_{m}$ is an affine function on $X$ such that

$$
g_{i} \leqq g^{\prime} \leqq h_{j} \quad(i=1, \cdots, m ; j=1, \cdots, n) .
$$

Hence the theorem applies with $f^{\prime}=\left.g^{\prime}\right|_{F^{\prime}}$ yielding $k_{1}$ such that $\left.k_{1}\right|_{F}=f$ and

$$
g_{i} \leqq k_{1} \leqq h_{j}+\frac{1}{2} \quad(i=1, \cdots, m ; j=1, \cdots, n) .
$$

Since $k_{1}-1 / 2, g_{i} \leqq h_{j}, k_{1}(i=1, \cdots, m ; j=1, \cdots, n)$ and $\left.\left(k_{1}-1 / 2\right)\right|_{F} \leqq$ $f=\left.k_{1}\right|_{F}$ the theorem applies again yielding $k_{2}$ such that 


$$
\begin{gathered}
\left\|k_{2}-k_{1}\right\| \leqq \frac{1}{2} \\
\left.k_{2}\right|_{F}=f \\
g_{i} \leqq k_{2} \leqq h_{j}+\frac{1}{4} \quad(i=1, \cdots, m ; j=1, \cdots, n) .
\end{gathered}
$$

Continuing by induction we get a sequence $\left\{k_{r}\right\}_{r=1}^{\infty}$ such that

$$
\begin{gathered}
\left\|k_{r+1}-k_{r}\right\| \leqq \frac{1}{2^{r}} \\
\left.k_{r}\right|_{F}=f \\
g_{i} \leqq k_{r} \leqq h_{j}+\frac{1}{2^{r}} .
\end{gathered}
$$

Hence $\left\{k_{r}\right\}_{r=1}^{\infty}$ converges to the desired $k$.

4. $\alpha$-polytopes. It was shown in [2] that the $\alpha$-polytopes [4] are conical at each closed face. We will show next that they are in fact decomposable at each closed face. Thus the (strongly) archimedean extension property holds at each face. This is a consequence of the fact that simplexes are decomposable at each closed face and the following theorem (see [2], Th. 3.7).

THEOREM 4.1. Let $X$ and $Y$ be compact convex sets whose closed faces span closed subspaces and let $\varphi: X \rightarrow Y$ be a continuous affine surjection. Let $\bar{\varphi}: A(X)^{*} \rightarrow A(Y)^{*}$ be the natural extension of $\varphi$ and suppose $\operatorname{dim}(\operatorname{ker} \bar{\varphi})<\infty$. If $F^{\prime \prime}$ is a closed face of $Y$ such that $X$ is decomposable at $\varphi^{-1}\left(F^{\prime}\right)$ then $Y$ is decomposable at $F^{\prime}$.

Proof. It is sufficient to consider the case where $\operatorname{ker} \bar{\varphi}=R x_{0}$. Let $N^{\prime}=\left\langle F^{\prime \prime}\right\rangle$ and $F=\phi^{-1}\left(F^{\prime}\right)$. Then $N \equiv\langle F\rangle=\bar{\varphi}^{-1}\left(N^{\prime}\right)+R x_{0}$. Let $f \in A(X)^{* *}$ such that $f \equiv 0$ on $N$ and

$$
X=\operatorname{conv}(F \cup\{x \in X: f(x) \geqq 1\}) \text {. }
$$

If $f\left(x_{0}\right)=0$ then $f \cdot \bar{\varphi}^{-1}$ well-defines a decomposing functional for $Y$ at $F^{\prime \prime}$. Suppose $f\left(x_{0}\right)=1$. Then $\left(x_{0}+N\right) \cap X=\dot{s}$ since if $x_{0}+n=$ $y \in X$ then $\varphi y=\bar{\varphi} n \in(\bar{\varphi} N) \cap Y=N^{\prime} \cap Y=F^{\prime}$ and hence $y \in \varphi^{-1}\left(F^{\prime \prime}\right)=$ $F$. But then $f(y)=f(n)=0$ contradicting $f\left(x_{0}\right)=1$. Thus there exists $r>0$ such that

$$
\left\|y-x_{0}+n\right\| \geqq r ; \text { for all } n \in N, x \in X \text {. }
$$

We define the bounded projection $p: A(X)^{*} \rightarrow f^{-1}(0)$ by $p(x)=x-f(x) x_{0}$. If $B_{r}=\left\{x \in A(X)^{*}:\|x\|<r\right\}$ then

$$
\left(N+B_{r}\right) \cap p(\{x \in X: f(x) \geqq 1\})=\dot{\phi} .
$$


If not there is $z \in X, f(z) \geqq 1$ and $n \in N$ such that $\|p(z)-n\|<r$. Let $x \in F$ and let $y \in X$ be given by

$$
y=(1 / f(z)) z+(1-1 / f(z)) x .
$$

Then

$$
\begin{aligned}
p(z)-n & =z-f(z) x_{0}-n=f(z) y-(f(z)-1) x-f(z) x_{0}-n \\
& =f(z)\left(y-x_{0}+n^{\prime}\right) .
\end{aligned}
$$

Thus $\left\|y-x_{0}+n^{\prime}\right\|<r / f(z) \leqq r$ contradicting (1).

Applying the separation theorem to (2) we obtain a bounded linear functional $g$ on $f^{-1}(0)$ such that $\|g\| \leqq 1 / r ; g \equiv 0$ on $N$ and $g \geqq 1$ on $p(\{x \in X: f(x) \geqq 1\})$. Thus $p X$ is decomposable at $p F=F^{r}$ under $g$ and $\bar{g}=g \cdot p \cdot \bar{\varphi}^{-1}$ decomposes $Y$ at $F^{\prime \prime}$.

5. Examples. We now give some elementary examples indicating the relationships between conical, decomposing and archimedean faces. Let $X$ be the closed convex set in the plane consisting of the unit square together with the disk $(x-1 / 2)^{2}+y^{2} \leqq 1 / 4$. Let $F$ be the face consisting of the line segment from $(0,0)$ to $(0,1)$. Then $X$ is not decomposable at $F$ since the only possible decomposing functional $(a, b) \rightarrow a$ does not work. Also $F$ is not an archimedean face of $X$ since the functional $(0, r) \rightarrow r$ on $F$ cannot be extended nonnegatively to $X$. On the other hand $X$ is conical at $F$ under the functional $(a, b) \rightarrow a$.

It is possible for $F$ to be archimedean without being a decomposing face. For example let $X$ be the intersection in the plane of $(x-1 / 2)^{2}+y^{2} \leqq 1 / 4$ and $x^{2}+(y-1 / 2)^{2} \leqq 1 / 4$ and let $F$ be the extreme point $\{(0,0)\}$. Then $X$ is not decomposable at $\{(0,0)\}$ or even conical there since these notations coincide for $F$ an extreme point. However $X$ is archimedean at $\{(0,0)\}$.

We next give an example of a closed face which is not selfdetermining. Let $S$ be the set of nonnegative sequences in $l^{1}$ with norm less than or equal to one (weak* topology as dual of $c_{0}$ ). Let $N$ be the subspace of sequences whose sum is zero and let $F$ be a norm compact convex subset of the unit ball (containing 0) such that the norm-closed linear span of $F$ is $N$. (Since $N$ is separable there is a sequence $\left(x_{n}\right)$ in $N$ such that $\left\|x_{n}\right\| \rightarrow 0$ and $N=\left\langle x_{n}\right\rangle^{-}$. Let $F=$ norm cl-conv $\left(x_{n}\right)$. Then $F$ is norm compact by Krein's Theorem.) Let $X=\operatorname{conv}(F \cup S)$. Since $S \subset X, A(X)$ consists exactly of all sequences in $c_{0}$ and their translates. But then $F$ is a closed face of $X$ whose linear span $N$ is weak* dense in $l^{1}$. Hence $\langle F\rangle^{-} \cap X=X$. However $X$ is nearly decomposable at $F$ in the sense that the bounded 
linear function $f$ on $l^{1}$ defined by $f(x)=\sum_{n=1}^{\infty} x_{n}$ is identically zero on $F$ and

$$
X=\operatorname{conv}(F \cup\{x \in X: f(x)=1\}) \text {. }
$$

\section{REFERENCES}

1. E. M. Alfsen, Facial structure of compact convex sets, Proc. London Math. Soc.

(3) 18 (1968), 385-404.

2. L. Asimow, Directed Banach spaces of affine functions, Trans. Amer. Math. Soc. 143 (1969), 117-132.

3. D. A. Edwards, Séparation des fonctions réelles définies sur un simplexe de Choquet, Comptes Rendus 261 (1965), 2798-2800.

4. R. R. Phelps, Infinite dimensional compact convex polytopes, Math. Scand. (to appear)

5. E. St $\phi$ rmer, On partially ordered vector spaces and their duals, with applications to simplexes and $C^{*}$-algebras, Proc. London Math. Soc. (3) 18 (1968), 245-265.

Received August 25, 1969. This work was partially supported by NSF Grant GP-11475.

University of California, Los ANGeles 



\section{PACIFIC JOURNAL OF MATHEMATICS}

\section{EDITORS}

H. SAmelson

Stanford University

Stanford, California 94305

RICHARd PieRCe

University of Washington

Seattle, Washington 98105

\author{
J. DugundJI \\ Department of Mathematics \\ University of Southern California \\ Los Angeles, California 90007 \\ RICHARD ARENS \\ University of California \\ Los Angeles, California 90024
}

ASSOCIATE EDITORS
E. F. BECKENBACH
B. H. NeumanN
F. WOLF
K. YosHIDA

\section{SUPPORTING INSTITUTIONS}

UNIVERSITY OF BRITISH COLUMBIA

CALIFORNIA INSTITUTE OF TECHNOLOGY

UNIVERSITY OF CALIFORNIA

MONTANA STATE UNIVERSITY

UNIVERSITY OF NEVADA

NEW MEXICO STATE UNIVERSITY

OREGON STATE UNIVERSITY

UNIVERSITY OF OREGON

OSAKA UNIVERSITY

UNIVERSITY OF SOUTHERN CALIFORNIA
STANFORD UNIVERSITY

UNIVERSITY OF TOKYO

UNIVERSITY OF UTAH

WASHINGTON STATE UNIVERSITY

UNIVERSITY OF WASHINGTON

AMERICAN MATHEMATICAL SOCIETY CHEVRON RESEARCH CORPORATION TRW SYSTEMS

NAVAL WEAPONS CENTER 


\section{Pacific Journal of Mathematics}

\section{Vol. 35, No. $1 \quad$ September, 1970}

B. D. Arendt and C. J. Stuth, On the structure of commutative periodic semigroups ..................................... 1

B. D. Arendt and C. J. Stuth, On partial homomorphisms of semigroups .... 7

Leonard Asimow, Extensions of continuous affine functions ............ 11

Claude Elias Billigheimer, Regular boundary problems for a five-term recurrence relation ................................... 23

Edwin Ogilvie Buchman and F. A. Valentine, A characterization of the

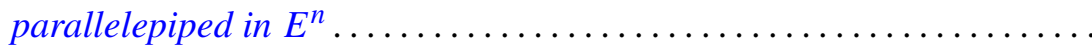
53

Victor P. Camillo, A note on commutative injective rings ............ 59

Larry Jean Cummings, Decomposable symmetric tensors ............. 65

J. E. H. Elliott, On matrices with a restricted number of diagonal values ... 79

Garth Ian Gaudry, Bad behavior and inclusion results for multipliers of type

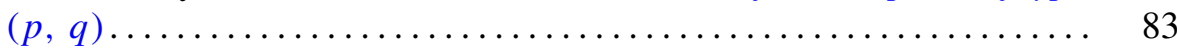

Frances F. Gulick, Derivations and actions .................. 95

Langdon Frank Harris, On subgroups of prime power index . . . . . . . . 117

Jutta Hausen, The hypo residuum of the automorphism group of an abelian

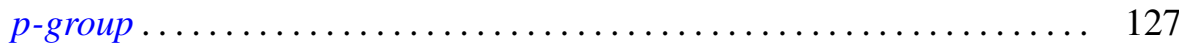

R. Hrycay, Noncontinuous multifuctions . ................... 141

A. Jeanne LaDuke, On a certain generalization of $p$ spaces ............ 155

Marion-Josephine Lim, Rank preservers of skew-symmetric matrices...... 169

John Hathway Lindsey, II, On a six dimensional projective representation of the Hall-Janko group ................................... 175

Roger McCann, Transversally perturbed planar dynamical systems . . . . . 187

Theodore Windle Palmer, Real $C^{*}$-algebras ................... 195

Don David Porter, Symplectic bordism, Stiefel-Whitney numbers, and a

Novikov resolution ....................................... 205

Tilak Raj Prabhakar, On a set of polynomials suggested by Laguerre

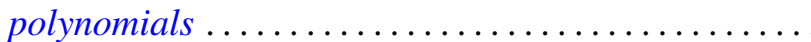

B. L. S. Prakasa Rao, Infinitely divisible characteristic functionals on locally convex topological vector spaces...................

John Robert Reay, Caratheodory theorems in convex product structures ...

Allan M. Sinclair, Eigenvalues in the boundary of the numerical range. .

David R. Stone, Torsion-free and divisible modules over matrix rings ....

William Jennings Wickless, A characterization of the nil radical of a

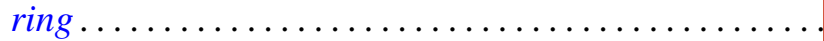

